\title{
The Comparison of the Edge Detection Methods in the Determination of Yarn Hairiness through Image Processing
}

\author{
Abdurrahman TELLI (iD) 0000-0002-6720-9410 \\ Cukurova University, Department of Textile Engineering, Adana, Turkey
}

Corresponding Author: Abdurrahman Telli, atelli@cu.edu.tr

\begin{abstract}
The resolution, quality and speed of the cameras have improved enormously in recent years. The combination of camera advancements and the software industry offers significant opportunities. In this study, an image processing approach for the determination of yarn hairiness was presented. Yarn images taken under a microscope were examined in MATLAB software. Seven different edge detection algorithms were used in order to separate the hairs from the yarn body. Seven different textural properties of obtained yarn images were compared with Zweigle hairiness test results. The findings have indicated that yarn hairiness can be clearly detected from microscope images with a six-step algorithm. The first four phases are grayscale, double format, 2D median filtering and histogram-fitting, respectively. The fifth stage is the edge detection algorithm and the sixth stage is the use of textural parameters. When compared with the Zweigle hairiness results, the most obvious finding to emerge from this study is that the best appropriate technique for edge detection was the Sobel method, and the textural parameter to be used in the evaluation was the standard deviation of matrix elements.
\end{abstract}

\author{
ARTICLE HISTORY \\ Received: 16.07 .2020 \\ Accepted: 09.06.2021
}

\section{KEYWORDS}

Yarn hairiness, determination of yarn hairiness, MATLAB, image processing, edge detection

\section{INTRODUCTION}

Image processing studies have gained importance in the characterization of textile materials in recent years. The objective test parameters obtained from fibers, yarns, fabrics, garments, carpets or composites in these studies are used in the various evaluation systems. It is seen that image processing studies have especially focused on defect and quality detection $[1,2]$. Yarn hairiness is an essential quality parameter for production processes and finished goods. It is defined as the protruding hairs from a yarn body. Commercially existing hairiness measurement systems have been used for a long time. There are basically two measurement methods used either a linear array of optical sensors or light scattering principles. However, these existing instruments do not meet the expectations. Previous studies have reported that instruments are not correctly measuring the real hairiness of spun yarns [3, 4]. Haleem and Wang (2015) have explained and discussed the limitations of these available hairiness measurement systems [5]. There were several studies to integrate existing yarn hairiness measurements with digital image and signal processing techniques $[6,7]$.

In the literature, academic researches containing serious discussions and analyses were realized based on image processing for the determination of yarn hairiness. For instance, a line-scan camera and computer to obtain a yarn acquisition system were used by Jackson et al., 1995. They implemented Fourier transforms in software. It has been identified as a normalised hairiness index [8]. Cybulska (1999) expressed yarns as divided into two structures (core and hairiness). Author emphasized that these structures should be evaluated independently. Hairiness was defined with the relationship between the areas of hairiness and yarn core [9]. Kuzanski has emphasized that yarn hairiness is formed by protruding fiber ends and the looped fibers. Author has made a construction of a research stand

To cite this article: Telli A. 2021. The Comparison of the Edge Detection Methods in the Determination of Yarn Hairiness through Image Processing. Tekstil ve Konfeksiyon, 31(2), 91-98. 
containing a microscope for yarn hairiness analysis in computer [10]. Kuzański and Jackowska-Strumiłło (2007) have shown the importance of isolating the fibre diameter itself to take the measurement protruding hairs from the yarn body. The authors proposed their algorithm of the edge detection in the yarn hairiness determination [11]. Fabijańska et al. (2008) applied the morphological opening and the dilation operations on yarn images obtained from the microscope [12]. Fabijańska (2010) discussed the best thresholding method for detecting protruding single fibers from the yarn body in considered class of images [13]. Fabijańska (2011) proposed a region growing algorithm for hairiness determination from images [14]. Fabijańska and Jackowska-Strumiłło (2012) have developed algorithms consisting of four main steps [15]. Guha et al. (2010) have remarked that true yarn hairiness can only be determined by examining the yarn under a microscope. Authors have emphasized that detection of the edge was an important point in determining the length of hairs. The identification of the edge was realized by using Canny's edge detection algorithm [16]. Roy et al. (2014) have installed a system containing cameras for image acquisition. The authors used the Sobel filter for yarn core segmentation [17]. Wang et al. (2014) have studied to solve the off-focus problem of hairy fibers protruding beyond the depth of field of the imaging system by applying a multifocus image fusion [18]. Gonçalves et al. (2015) used logical operations (XOR) to isolate and quantify the protruding and loop fibers from the yarn core [19]. Sengupta et al. (2015) presented an algorithm including nine steps in their study [20]. Li et al. (2016) proposed an image acquisition and processing system with a video camera to evaluate yarn appearance. Formation of a digital yarn blackboard was ensured by capturing sequence images of a moving yarn and extracting yarn diameter data after the image threshold and morphological opening operation [21]. Wang et al. (2021) scanned images of original yarn blackboard. The yarn body and protruding fibers were segmented from the blackboard background with algorithms to extract the index for yarn evenness and hairiness [22]. In some studies, CCD sensors were used. CCDs are image sensors used in video cameras. It records the light entering the camera by converting it into electronic signals [23-24]. A CCD line scan sensor by Pan et al. (2018) and two CCD sensors by Li et al. (2019) were used for their developed yarn cross-sectional analysis system. Then, researchers were validated of their results with existing commercial yarn measurement systems. Li et al. (2019) emphasized that the yarn transfer speeds must be optimized as the yarn traverses between the CCD sensors. Researchers have stated that the image resolution changes with a change in yarn delivery speed and the results of hairiness in the image reduce as the delivery speed is increased [24].

The existing hairiness measurement systems measure by making a 90 degree angle with the yarn in the direction of vertical or horizontal. However, the placement of the protruding fibers on the main body has a unique structure. There is no 90 degree angle. There is a layout at different angles. Due to this special structure, the angular placement of the hairs changes with any external effect (speed, air, contact...). Therefore, lengths of hairs on opposite sides of the camera and the back of the yarn determine incorrectly. To solve this problem, Wang et al. (2018) used two angled planar mirrors to view a yarn from five different perspectives simultaneously, and a digital camera to capture the multiple images in one panoramic picture [25]. Wang et al. (2019) acquired yarn images from different viewing angles by rotating yarns as 15 degree by the CMOS camera. Length and number of yarn hairiness obtained from different viewing angles were calculated to evaluate hairiness results [26]. In general, it is seen that the studies in the literature cannot offer the speed provided by the current hairiness measurement methods. When the desired speed is reached with signal processing techniques, it gives inconsistent results as in other methods. However, new studies provide important clues for revising existing methods. In earlier studies, microscope examinations were used as it provides a real magnified image in the yarn appearance evaluations. However, the image obtained under the microscope was losing its quality while being transferred to the computer because the resolutions of the cameras were low. The resolution, quality and speed of the cameras have improved enormously in recent years. The combination of camera advancements and the software industry offers significant opportunities. It started to be used in many areas such as license plate recognition, face recognition, body measurements in apparel and pattern making.

In this study, real images, which were magnified 10 times under a microscope and transferred to a computer with high resolution and quality, were used. It has been tried to produce numerical data from the obtained images with the most appropriate algorithms. Seven different edge detection algorithms were investigated. Differently from literature, textural properties of yarn images were examined in MATLAB software. The results were compared with the Zweigle hairiness test results.

\section{MATERIAL AND METHOD}

$100 \%$ Cotton Ne 30 carded ring, combed ring and rotor yarns showing different hairiness properties were used in this research. Images of yarns were taken at 10x (10-fold) magnification ratio using a stereomicroscope of the Novel NSZ 808 brand for surface digitization. Ten different images were taken from each yarn with approximately 10 meters intervals from 100 meters. The hairiness properties of these three different yarns were tested using Zweigle $G$ 567 with five specimens and 100 meters per yarn type with the testing speed of $50 \mathrm{~m} / \mathrm{mn}$. As hairiness properties can change with the effect of external factors, microscope analysis and hairiness tests were carried out independently of each other. Tests and analysis were made at standard atmospheric conditions $\left(20 \pm 2{ }^{\circ} \mathrm{C}\right.$ temperature and $\% 65 \pm 4$ relative humidity) after conditioning according to TS EN ISO 139. 


\subsection{Image Processing}

MATLAB R2018a software was used for image processing. Firstly, yarn images in RGB color format (1000x 2500x3 uint8) taken from microscope were loaded to MATLAB. Images in RGB color format taken from microscope were transformed grayscale to be processed in following steps. After, these matrices in "1000x2500 uint8" format were transformed to double $(1000 \times 2500$ double $)$ format. A median filter was implemented on matrices in this format and image intensity values were adjusted for edge detection by using histogram-fitting $[27,28]$. Then, edge detection was applied in seven different methods. These methods are 'Sobel', 'Prewitt', 'Roberts', 'log', 'zerocross', 'Canny' and 'approxcanny'. For the gradient-magnitude edge detection methods (Sobel, Prewitt, and Roberts), edge uses threshold to threshold the calculated gradient magnitude. For the zero-crossing methods, including Laplacian of Gaussian (LoG), edge uses threshold as a threshold for the zero-crossings. The Canny method applies two thresholds to the gradient: a high threshold for low edge sensitivity and a low threshold for high edge sensitivity. Calculated threshold value used in the computation, returned as a 2element vector for the 'Canny' method, an empty vector ([]) for the 'approxcanny' method, or a numeric scalar for all other edge detection methods [29].

Seven statistical measures extracting from matrices were investigated to characterize the textural properties of obtained images after edge detection methods. These are mean of matrix elements, the standard deviation of matrix elements, the entropy of grayscale images, contrast, correlation, energy and homogeneity. Contrast, correlation, energy, and homogeneity are statistical properties of the image derived from the gray level co-formation matrix (GLCM). Contrast measures regional variations in GLCM. The correlation tests the combined probability of the specified pairs of pixels. Energy gives the sum of the square elements in the GLCM. Homogeneity measures the proximity of the distribution of elements in the GLCM to the GLCM diagonal. The mean of the matrix elements is the average of all values in the array. The standard deviation of the matrix elements is the standard deviation of the pixel density values. Entropy is related to normalized histogram numbers. It is easily possible to reach from an open-access MATLAB database to the explanations and details related to the used edge detection methods and the performed functions for textural properties [27-28, 30-33].

For the mentioned image processing steps, it is necessary to enter a large number of code lines in the MATLAB command window in the first generation of the algorithm. For example, a summary of the used code lines was given below in the image processing steps for the first sample of the carded ring yarn from 30 different images taken from three different yarn types.

$\%$ To convert images uploaded to MATLAB software to Grayscale format (1st stage)
>> AxKardex01=rgb2gray (xKardex01);

$\%$ To convert Grayscale format to double format (2nd stage)

> ADxKardex01=im2double $($ AxKardex01);

$\%$ Application of 2D Median Filtering (3rd stage)

> MADxKardex01=medfilt2 (ADxKardex01);

$\%$ Application of Histogram-Fitting (4th stage)

> JMADxKardex01=imadjust (MADxKardex01);

$\%$ Application of Edge-Detection Methods (5th stage)

>> CANNYxKardex01=edge (JMADxKardex01, 'Canny');

> ZerocrossxKardex01=edge (JMADxKardex01, 'zerocross');

> ApproxcannyxKardex01=edge (JMADxKardex01, 'approxcanny');

>> LogxKardex01=edge (JMADxKardex01, 'log');

> PrewittxKardex01=edge (JMADxKardex01, 'Prewitt');

>> SobelxKardex01=edge (JMADxKardex01, 'Sobel');

>> RobertsxKardex01=edge (JMADxKardex01, 'Roberts');

$\%$ To extract seven statistical measures from matrices(6th stage)

$\%$ The extraction of three parameters (mean of matrix elements, the standard deviation of matrix elements, the entropy of grayscale images)

> SxPrewittxKardex01=std2 (PrewittxKardex01);

>> SxSobelxKardex01=std2 $($ SobelxKardex01);

> SxRobertsxKardex01=std2 (RobertsxKardex01);

> MxPrewittxKardex01=mean2 (PrewittxKardex01);

> MxSobelxKardex01=mean2 (SobelxKardex01);

>> MxRobertsxKardex01=mean2 (RobertsxKardex01);

> ExPrewittxKardex01=entropy (PrewittxKardex01);

> ExSobelxKardex01=entropy (SobelxKardex01);

> ExRobertsxKardex01=entropy (RobertsxKardex01);

$\%$ The extraction of four parameters derived from the gray level co-formation matrix (Contrast, correlation, energy, and homogeneity)

>> GxPrewittxKardex01=graycomatrix(PrewittxKardex01);

> GxSobelxKardex01=graycomatrix(SobelxKardex01);

>> GxRobertsxKardex01=graycomatrix(RobertsxKardex01);

> StatsxPrewittxKardex01=graycoprops $($ GxPrewittxKardex01);

>> StatsxSobelxKardex01=graycoprops $($ GxSobelxKardex01);

>> StatsxRobertsxKardex01=graycoprops(GxRobertsxKardex01);

The relationship between yarn hairiness test results and results of the measurement of textural properties were statistically evaluated using correlation analysis. The significance level (p) of variables obtained from the normality test is shown in Table 1 . 
Table 1. The significance level (p) of variables obtained from the normality test

\begin{tabular}{|c|c|c|c|c|c|c|c|c|c|c|}
\hline \multirow[b]{2}{*}{$\begin{array}{l}\text { Edge } \\
\text { Detection } \\
\text { Methods }\end{array}$} & \multicolumn{7}{|c|}{ Textural Properties } & \multicolumn{3}{|c|}{ Hairiness Properties } \\
\hline & $\begin{array}{c}\text { Mean of } \\
\text { matrix } \\
\text { elements }\end{array}$ & $\begin{array}{c}\text { Standard } \\
\text { deviation } \\
\text { of matrix } \\
\text { elements }\end{array}$ & $\begin{array}{l}\text { Entropy } \\
\text { of } \\
\text { grayscale } \\
\text { images }\end{array}$ & Contrast & Correlation & Energy & Homogeneity & $\mathrm{S} 3$ & $\mathrm{~S} 12$ & $\begin{array}{c}\text { S3 } \\
+ \\
\text { S12 }\end{array}$ \\
\hline Sobel & 0,443 & 0,626 & 0,545 & 0,463 & 0,870 & 0,320 & 0,200 & & & \\
\hline Prewitt & 0,543 & 0,619 & 0,530 & 0,385 & 0,853 & 0,349 & 0,453 & 0,677 & 0,366 & 0,397 \\
\hline Roberts & 0,799 & 0,915 & 0,845 & 0,584 & 0,858 & 0,381 & 0,654 & & & \\
\hline
\end{tabular}

Table 2.The mean test results of hairiness of yarns

\begin{tabular}{cccccccccccccc}
\hline No & Type & $\begin{array}{c}\mathbf{1} \\
\mathbf{m m}\end{array}$ & $\begin{array}{c}\mathbf{2} \\
\mathbf{m m}\end{array}$ & $\begin{array}{c}\mathbf{3} \\
\mathbf{m m}\end{array}$ & $\begin{array}{c}\mathbf{4} \\
\mathbf{m m}\end{array}$ & $\begin{array}{c}\mathbf{6} \\
\mathbf{m m}\end{array}$ & $\begin{array}{c}\mathbf{8} \\
\mathbf{m m}\end{array}$ & $\begin{array}{c}\mathbf{1 0} \\
\mathbf{m m}\end{array}$ & $\begin{array}{c}\mathbf{1 2} \\
\mathbf{m m}\end{array}$ & $\begin{array}{c}\mathbf{1 5} \\
\mathbf{m m}\end{array}$ & $\mathbf{S 3}$ & $\mathbf{S 1 2}$ & $\begin{array}{c}\mathbf{S 3} \\
\mathbf{S}\end{array}$ \\
\hline 01 & $\begin{array}{c}\text { Carded } \\
\text { Ring }\end{array}$ & 18367 & 6292 & 2049 & 1093 & 366 & 80 & 11 & 1 & 0 & 3599 & 24659 & 28258 \\
02 & $\begin{array}{c}\text { Combed } \\
\text { Ring }\end{array}$ & 15188 & 3505 & 986 & 477 & 122 & 22 & 3 & 0 & 0 & 1611 & 18693 & 20304 \\
03 & $\begin{array}{c}\text { Rotor } \\
\text { Yarn }\end{array}$ & 2737 & 607 & 215 & 141 & 41 & 8 & 1 & 0 & 0 & 406 & 3344 & 3749 \\
\hline
\end{tabular}

Since the variables were normally distributed, the degree of correlation between variables was determined by Pearson coefficient of correlation (r). When the correlation coefficient (r) value is between 0.75 and 1.00 , it means that there is a strong relationship.

\section{RESULTS AND DISCUSSION}

The mean results obtained from the Zweigle hairiness tests are shown in Table 2. It can be seen from the data in Table 2 that there were significant differences amongst yarns in terms of yarn hairiness. There were the highest hairiness values in carded ring yarns coded as "01" and the lowest hairiness values were found in rotor yarns coded as "03". This situation has been provided consciously by researchers with the choice of yarns from different spinning systems in the same yarn count to enrich the research through different hairiness levels.

To make the subject more understandable, images in process steps of the first sample of carded ring yarn from 30 different images taken from three different yarns are given respectively in Figure 1.

As mentioned in the material and methods section, there were fourstages before the edge detection methods. Images after seven different edge detection methods of the same sample can be compared in Figure 2.

When the images are evaluated visually, it is clearly seen from Figure 2 that Sobel, Prewitt and Roberts from edge detection methods reflects hairs separated from the yarn body better than others.In other images, separation of the hairs from the yarn body is seen as problematic. There is significant noise in the images of 'log', 'zerocross', 'Canny' and 'approxcanny' edge detection methods.For this reason, the images obtained from Sobel, Prewitt and Roberts methods were used in later stages. The mean results of seven different textural parameters extracted from matrices obtained from images of three methods are given in Table 3. Table
4 shows the obtained correlation coefficient (r) values between Zweigle yarn hairiness and these textural properties of images.

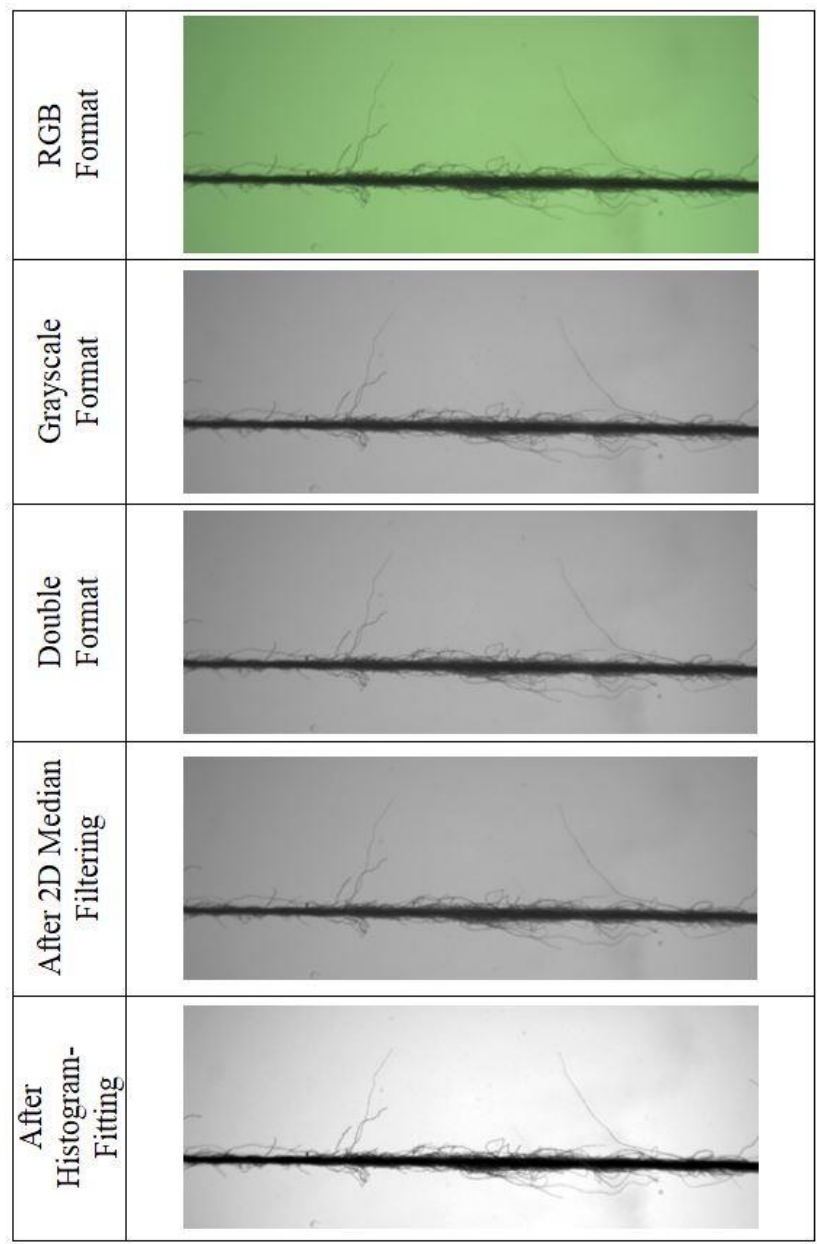

Figure 1.Images after process steps in image processing 
As examined the mean of matrix elements in Table 3, it was seen in all three methods that the highest values were measured in carded ring yarns coded as "01" and the lowest values were measured in rotor yarns coded as "03". According to the correlation results obtained from Table 4, there was a strong correlation between the mean of the matrix elements and Zweigle hairiness values in the Sobel and Prewitt methods.In the Roberts method, lower correlation values of less than 0.75 were observed for the mean of the matrix elements. The maximum correlation value between the mean of matrix elements and Zweigle hairiness was determined in $\mathrm{S} 3+\mathrm{S} 12$ hairiness with Sobel method $(r=0.790)$.

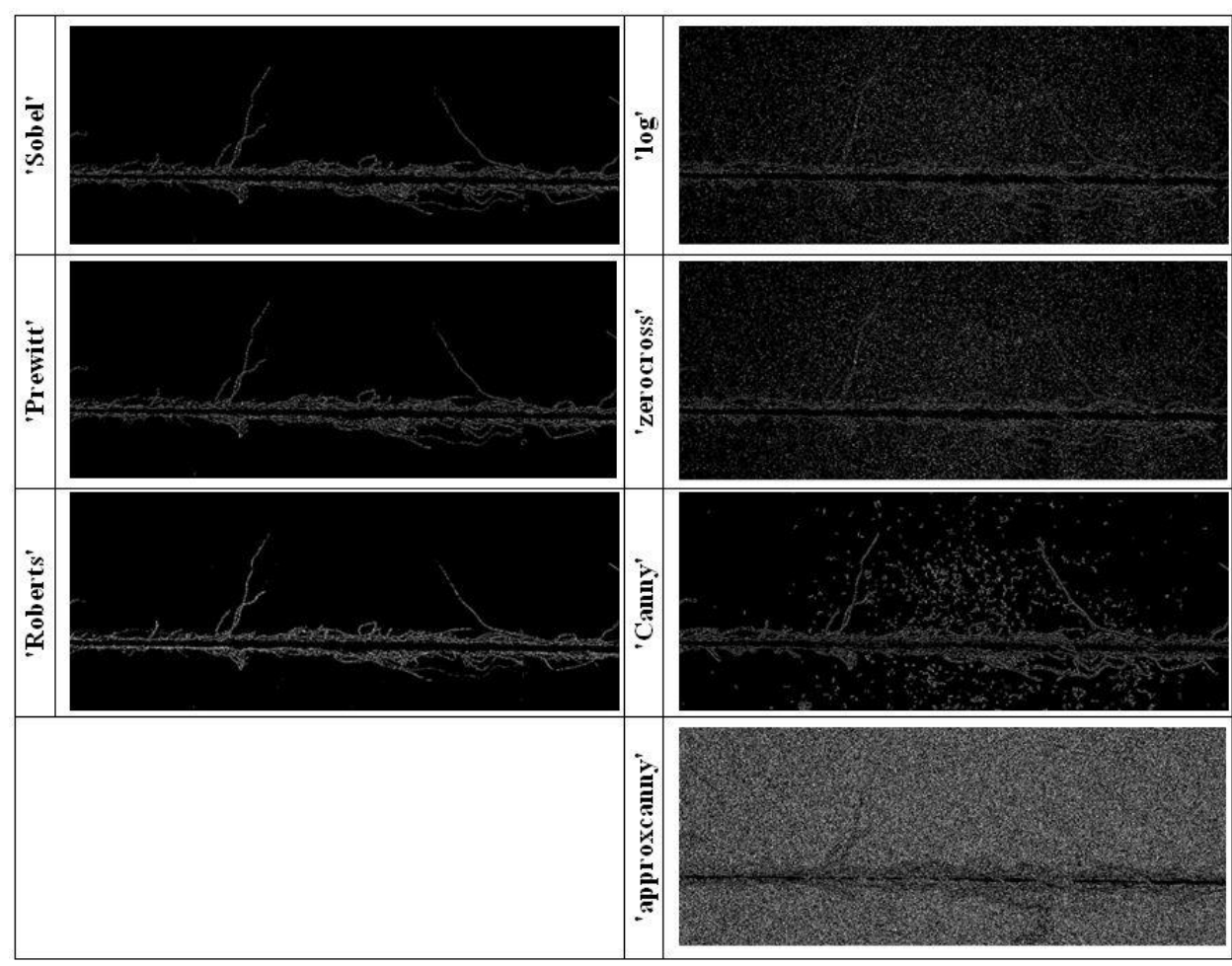

Figure 2.Images after edge detection methods

Table 3.The mean results of seven different textural parameters

\begin{tabular}{|c|c|c|c|c|c|c|c|c|c|}
\hline \multirow[b]{2}{*}{$\begin{array}{c}\text { Textural } \\
\text { Properties }\end{array}$} & \multicolumn{3}{|c|}{ Sobel } & \multicolumn{3}{|c|}{ Prewitt } & \multicolumn{3}{|c|}{ Roberts } \\
\hline & $\begin{array}{c}01 \\
\text { Carded } \\
\text { Ring }\end{array}$ & $\begin{array}{c}02 \\
\text { Combed } \\
\text { Ring }\end{array}$ & $\begin{array}{c}03 \\
\text { Rotor } \\
\text { Yarn }\end{array}$ & $\begin{array}{c}01 \\
\text { Carded } \\
\text { Ring }\end{array}$ & $\begin{array}{c}02 \\
\text { Combed } \\
\text { Ring }\end{array}$ & $\begin{array}{c}03 \\
\text { Rotor Yarn }\end{array}$ & $\begin{array}{c}01 \\
\text { Carded } \\
\text { Ring }\end{array}$ & $\begin{array}{c}02 \\
\text { Combed } \\
\text { Ring }\end{array}$ & $\begin{array}{c}03 \\
\text { Rotor } \\
\text { Yarn }\end{array}$ \\
\hline $\begin{array}{l}\text { Mean of } \\
\text { matrix } \\
\text { elements }\end{array}$ & 0,0122 & 0,0102 & 0,0086 & 0,0121 & 0,0102 & 0,0086 & 0,0153 & 0,0134 & 0,0117 \\
\hline $\begin{array}{l}\text { Standard } \\
\text { deviation of } \\
\text { matrix } \\
\text { elements }\end{array}$ & 0,1095 & 0,1005 & 0,0923 & 0,1092 & 0,1002 & 0,0921 & 0,1227 & 0,1149 & 0,1073 \\
\hline $\begin{array}{l}\text { Entropy of } \\
\text { grayscale } \\
\text { images }\end{array}$ & 0,0949 & 0,0823 & 0,0714 & 0,0945 & 0,0818 & 0,0712 & 0,1142 & 0,1025 & 0,0916 \\
\hline Contrast & 0,0097 & 0,0076 & 0,0071 & 0,0095 & 0,0074 & 0,0070 & 0,0106 & 0,0088 & 0,0083 \\
\hline Correlation & 0,5991 & 0,6227 & 0,5865 & 0,6051 & 0,6290 & 0,5925 & 0,6480 & 0,6674 & 0,6424 \\
\hline Energy & 0,9663 & 0,9722 & 0,9759 & 0,9666 & 0,9725 & 0,9761 & 0,9593 & 0,9649 & 0,9687 \\
\hline Homogeneity & 0,9919 & 0,9962 & 0,9965 & 0,9953 & 0,9963 & 0,9965 & 0,9947 & 0,9956 & 0,9959 \\
\hline
\end{tabular}


Table 4. The obtained correlation coefficient ( $r$ ) values between yarn hairiness and textural properties of images

\begin{tabular}{|c|c|c|c|c|c|c|c|c|c|}
\hline \multirow{2}{*}{$\begin{array}{c}\text { Textural } \\
\text { Properties }\end{array}$} & \multicolumn{3}{|c|}{ Sobel } & \multicolumn{3}{|c|}{ Prewitt } & \multicolumn{3}{|c|}{ Roberts } \\
\hline & S3 & S12 & S3+S12 & $\mathbf{S 3}$ & S12 & S3+S12 & $\mathbf{S 3}$ & S12 & $\mathrm{S} 3+\mathrm{S} 12$ \\
\hline $\begin{array}{l}\text { Mean of matrix } \\
\text { elements }\end{array}$ & 0.769 & 0.784 & 0.790 & 0.767 & 0.781 & 0.786 & 0.718 & 0.739 & 0.743 \\
\hline $\begin{array}{l}\text { Standard deviation } \\
\text { of matrix elements }\end{array}$ & 0.770 & 0.794 & 0.798* & 0.771 & 0.790 & 0.795 & 0.727 & 0.751 & 0.755 \\
\hline $\begin{array}{l}\text { Entropy of } \\
\text { grayscale images }\end{array}$ & 0.771 & 0.788 & 0.793 & 0.771 & 0.784 & 0.790 & 0.724 & 0.746 & 0.750 \\
\hline Contrast & 0.611 & 0.596 & 0.603 & 0.618 & 0.596 & 0.604 & 0.604 & 0.585 & 0.592 \\
\hline Correlation & 0.103 & 0.166 & 0.160 & 0.103 & 0.173 & 0.165 & 0.002 & 0.090 & 0.080 \\
\hline Energy & -0.747 & -0.753 & -0.759 & -0.749 & -0.749 & -0.756 & -0.706 & -0.714 & -0.720 \\
\hline Homogeneity & -0.614 & -0.602 & -0.609 & -0.617 & -0.595 & -0.603 & -0.597 & -0.576 & -0.584 \\
\hline
\end{tabular}

For all three methods, carded ring coded as "01" have the highest values in the standard deviation of matrix elements. The lowest values were found in rotor yarns coded as "03" (Table 3). The correlation results indicated thatthere is a strong correlation between the standard deviation of matrix elements and Zweigle hairiness values in the Sobel and Prewitt methods as in the results of the mean of matrix elements.As distinct from the mean of matrix elements, the findings in the Roberts method showed that there is a strong correlation between the standard deviation of matrix elements with S12 and S3 + S12. However, a lower correlation was observed in S3 hairiness (Table 4).S3 index is the total number of fibers in the length zones $\geq 3$ mm.However, the standard deviation of matrix elements is a textural expression and is related to the whole of the image. Therefore, all hairs separated from the yarn body are included in the calculation. When Table 4 is considered as a whole, it will be seen that S3 + S12 showed higher correlation values compared to S3 and S12 in all parameters where a strong correlation was observed. This is an expected situation since all the hairs separated from the yarn body are taken into account in the textural parameters.It can be seen from the data in Table 4 that the maximum correlation value between the standard deviation of matrix elements and Zweigle hairiness was determined in $\mathrm{S} 3+\mathrm{S} 12$ hairiness with the Sobel method $(\mathrm{r}=0,798)$.

As examined the entropy of grayscale images, it can be seen from Table 3 that the highest values were measured in carded ring yarns coded as "01" and the lowest values were measured in rotor yarns coded as "03" for all three methods.From the correlation results in Table 4, it is apparent that there was a strong correlation between the entropy of grayscale images and Zweigle hairiness values in the Sobel and Prewitt methods.It was found that there was a strong correlation between the entropy of grayscale images and only S3 + S12 in the findings in the Roberts method. The similar to the mean of matrix elements and the standard deviation of matrix elements, the maximum correlation value between the entropy of grayscale images and Zweigle hairiness was determined in S3+S12 hairiness with Sobel method $(r=0,793)$.

According to contrast results from Table 3, carded ring yarns coded as "01" have the highest values and rotor yarns coded as "03" have the lowest values in all methods. However, the correlation results from Table 4 showed that there was no strong correlation for contrast between Zweigle hairiness indices and all edge detection methods.As can be seen from Table 3, there was no trend in the correlation findings of textural parameters. Furthermore, no significant correlation coefficient (r) values were found between correlation findings of textural parameters and Zweigle hairiness indices (Table 4).

When the energy results for all three methods in Table 3 were examined, the highest values were observed in rotor yarns coded as "03". The lowest values were determined in carded ring yarns coded as "01". However, the trend of energy values was realized opposite to the results of mean of matrix elements, the standard deviation of matrix elements, and entropy of grayscale images. Looking at the correlation results in Table 4, it can be seen that there were negative correlations between energy results and the results of Zweigle hairiness indices in all three methods. The correlation results indicated that there was no strong correlation between energy and Zweigle hairiness indices in Roberts's method. In the Prewitt method, there was a negative significant correlation with $\mathrm{S} 3+\mathrm{S} 12$ hairiness. The findings in the Sobel method showed that there was a negative significant correlation between energy values with $\mathrm{S} 12$ and S3 + S12. Furthermore, the correlation coefficient values in the Sobel between energy and S3 + S12 was higher than the Prewitt method. As can be seen from Table 4 , the maximum correlation value between energy and Zweigle hairiness indices was found in $\mathrm{S} 3+\mathrm{S} 12$ hairiness index with the Sobel method ( $\mathrm{r}=-0.759)$.

Similar to the energy results in terms of homogeneity, rotor yarns coded as "03" have the highest values and carded ring yarns coded as "01" have the lowest values amongst all methods (Table 3). The trend of homogeneity values was 
realized opposite to the results of mean of matrix elements, standard deviation of matrix elements, and entropy of grayscale images. Furthermore, it was observed in Table 4 that there were negative correlations between homogeneity results and the results of Zweigle hairiness indices in all three methods similarly to the situation of energy values.However,no significant correlation coefficient (r) values were determined between homogeneity findings of textural parameters and Zweigle hairiness indices (Table 4).

In summary, previous works have used different edge detection methods to find yarn body in the determination of yarn hairiness using image processing $[16,17]$. Guha et al. (2010) suggested Canny's edge detection algorithmfor yarn body segmentation [16]. However, it can be seen from the images after edge detection methods shown in Figure 2 that Sobel, Prewitt and Roberts reflect hairs separated from the yarn body better than Canny and others. Roy et al. (2014) proposed Sobel algorithm in their method [17]. The findings of the current study support their research containing the Sobel algorithm. The higher correlation values were obtained in the Sobel method between the textural parameters and the Zweigle hairiness indices than other edge detection methods. In addition to Sobel, Prewitt method also showed higher correlation values than others in this study.In general in this study, the highest correlation $(\mathrm{r}=0.798)$ was observed in the Sobel method between standard deviation of matrix elements and $\mathrm{S} 3+\mathrm{S} 12$ hairiness in the relationship between yarn hairiness and textural properties of images. The highest correlation in S12 hairiness was also measured in Sobel method with standard deviation of matrix elements $(r=0.794)$. The highest correlation between S3 hairiness and textural parameters was obtained in three different situations. Entropy of grayscale images in Sobel method, entropy of grayscale images in Prewitt method, and the standard deviation of matrix elements in Prewitt method showed the same correlation results $(\mathrm{r}=0.771)$.

Mean of matrix elements, standard deviation of matrix elements and entropy of grayscale imagesfrom textural parameters were used in a limited number of previous studies. Telli (2019) and Telli (2020) indicated that success has been achieved by using means of matrix elements in their developed method to determine the pilling grade of fabrics [27-28]. In this study, these three textural parameters showed strong correlation over 0.75 with all Zweigle indices in the Sobel and Prewitt methods. The present findings seem to be consistent with other research. Contrast, correlation, energy and homogeneity were proposed by Haralick et al. (1973) to identify the texture of images [34]. These four texture features based on cooccurrence matrix were investigated in numerous prior studies [2, 35, 36].In the subjective assessment of pilling resistance of woven fabrics, Eldessouki and Hassan (2015) have proposed only the usage of contrast and correlation from the four relevant properties due to their repeatable behavior and opposite trends [36].In opposition to earlier findings, however, no relationship of contrast and correlation was detected with hairiness. Negative strong correlation values were only obtained in energy from four texture features. In Sobel ( $\mathrm{r}=-0.759)$ and Prewitt $(\mathrm{r}=-0.756)$ methods, there is a strong negative correlation with S3 + S12 hairiness. A strong negative correlation was found with $\mathrm{S} 12$ hairiness in Sobel method $(\mathrm{r}=-0.753)$.

\section{CONCLUSION}

This study was opened by noting that conventional measurement methods of yarn hairiness are not correctly measuring the real hairiness of spun yarns. Devices are considered to be inadequate due to the new developed yarn types, different production methods and better quality expectations in recent years. It is known that current methods do not provide sensitive measuring. Instruments determine general results and obvious differences. Their advantages are that they offer a continuous and fast assessment. The vast majority of the literature is related to revising existing techniques to be more efficient. In this investigation, the aim was to test real hairiness results by using real yarn images taken under microscope differently from signal processing and optical measurements. The advancements in camera and the software industry in recent years give us this significant opportunity.

The findings of this research provide important contributions to the literature. The findings have indicated that yarn hairiness can be clearly detected from microscope images with a six-step algorithm. The first four phases are grayscale, double format, 2D median filtering and histogram-fitting, respectively. The fifth stage is the edge detection algorithm and the sixth stage is the use of textural parameters. When compared with the Zweigle hairiness results, the most obvious finding to emerge from this study is that the best appropriate technique for edge detection was the Sobel method, and the textural parameter to be used in the evaluation was the standard deviation of matrix elements. Feeding a large number of data to the software to be developed using the six-step image processing technique proposed in this study will open the way to obtain more accurate and comprehensive hairiness results.

The major limitation of this study is that the image acquisition step is static. It is necessary to acquire a dynamic structure with a device that will provide yarn flow and a stereomicroscope with high resolution and speed if it is desired to transition to commercial applications. Thus, more reliable information about the whole yarn can be obtained when the system is developed. In future studies, the scope of the study can be expanded with different yarn counts and fiber blends. Their results can be compared with the results of other commercial devices apart from Zweigle indices. 


\section{REFERENCES}

1. Behera, B. K. (2004). Image-processing in textiles. Textile Progress 35(2-4), 1-193.

2. Wong, C. (Ed.). (2017). Applications of Computer Vision in Fashion and Textiles. Woodhead Publishing.

3. Haleem, N., \& Wang, X. (2013). A comparative study on yarn hairiness results from manual test and two commercial hairiness metres. Journal of The Textile Institute, 104(5), 494-501

4. Zhang, G., \& Xin, B. (2016). An overview of the application of image processing technology for yarn hairiness evaluation. Research Journal of Textile and Apparel. 20(1), 24-36.

5. Haleem, N., \& Wang, X. (2015). Recent research and developments on yarn hairiness. Textile Research Journal, 85(2), 211-224.

6. Carvalho, V., Cardoso, P., Belsley, M., Vasconcelos, R. M. \&Soares, F. O. (2008). Yarn hairiness parameterization using a coherent signal processing technique. Sensors and Actuators A: Physical, 142(1), 217-224.

7. Ozkaya, Y. A., Acar, M., \& Jackson, M. R. (2008). Simulation of photosensor-based hairiness measurement using digital image analysis. The Journal of The Textile Institute, 99(2), 93-100.

8. Jackson, M., Acar, M., Yuen, S. L., \&Whitby, D. (1995). A vision based yarn scanning system. Mechatronics, 5(2-3), 133-146.

9. Cybulska, M. (1999). Assessing yarn structure with image analysis methods1. Textile Research Journal, 69(5), 369-373.

10. Kuzanski, M. (2006, May). Measurement Methods for Yarn Hairiness Analysis-the idea and construction of research standing. In Proceedings of the 2nd international conference on perspective technologies and methods in MEMS design (pp. 87-90). IEEE.

11. Kuzanski, M., \&Jackowska-Strumillo, L. (2007, May). Yarn hairiness determination the algorithms of computer measurement methods. In 2007 International Conference on Perspective Technologies and Methods in MEMS Design (pp. 155-158). IEEE.

12. Fabijanska, A., Kuzanski, M., Sankowski, D., \&JackowskaStrumillo, L. (2008, May). Application of image processing and analysis in selected industrial computer vision systems. In 2008 International Conference on Perspective Technologies and Methods in MEMS Design (pp. 27-31). IEEE.

13. Fabijańska, A. (2010, April). A survey of thresholding algorithms on yarn images. In 2010 Proceedings of VIth International Conference on Perspective Technologies and Methods in MEMS Design (pp. 23 26). IEEE.

14. Fabijańska, A. (2011). Yarn image segmentation using the region growing algorithm. Measurement Science and Technology, 22(11) 114024.

15. Fabijańska, A., \&Jackowska-Strumiłło, L. (2012). Image processing and analysis algorithms for yarn hairiness determination. Machine Vision and Applications, 23(3), 527-540.

16. Guha, A., Amarnath, C., Pateria, S., \& Mittal, R. (2010) Measurement of yarn hairiness by digital image processing. The journal of the Textile Institute, 101(3), 214-222.

17. Roy, S., Sengupta, A., \&Sengupta, S. (2014). Yarn hairiness evaluation using image processing. In Proceedings of The 2014 International Conference on Control, Instrumentation, Energy and Communication (CIEC) (pp. 588-592). IEEE.

18. Wang, R., Zhou, J., Yu, L., \& Xu, B. (2014). Fusing multifocus images for yarn hairiness measurement. Optical Engineering, 53(12), 123101.

19. Goncalves, N., Carvalho, V., Belsley, M., Vasconcelos, R. M., Soares, F. O., \& Machado, J. (2015). Yarn features extraction using image processing and computer vision-A study with cotton and polyester yarns. Measurement, 68, 1-15.
20. Sengupta, A., Roy, S., \&Sengupta, S. (2015). Development of a low cost yarn parameterisation unit by image processing. Measurement, 59, 96-109.

21. Li, Z., Pan, R., \&Gao, W. (2016). Formation of digital yarn black board using sequence images. Textile Research Journal, 86(6), 593603

22. Wang, L., Lu, Y., Pan, R., \&Gao, W. (2021). Evaluation of yarn appearance on a blackboard based on image processing. Textile Research Journal, DOI: 10.1177/00405175211002863.

23. Pan, Q., Chen, M., Hu, Y., \&Zuo, B. (2018). System design for evenness measurement of raw silk. Fibres \& Textiles in Eastern Europe.26, 1(127): 21-24. DOI: 10.5604/01.3001.0010.7790

24. Li, G., Akankwasa, N. T., Zhao, Q., \& Wang, J. (2019). A Novel System for Yarn Cross-Section Analysis Based on Dual Orthogonal CCD Sensors. Journal of Natural Fibers, 16(1), 114-125.

25. Wang, L., Xu, B., \&Gao, W. (2018). Multi-perspective measurement of yarn hairiness using mirrored images. Textile Research Journal, 88(6), 621-629.

26. Wang, W., Xin, B., Deng, N., \& Li, J. (2019). Objective evaluation on yarn hairiness detection based on multi-view imaging and processing method. Measurement, 148, 106905.

27. Telli, A. (2019). An Image Processing Research Consistent with Standard Photographs to Determine Pilling Grade of Woven Fabrics. TekstilveKonfeksiyon, 29(3), 268-276.

28. Telli, A. (2020). The Relationship Between Subjective Pilling Evaluation Results and Detecting Pills and Textural Features in Knitted Fabrics. Fibers and Polymers, 21(8), 1841-1848.

29. Mathworks, Find edges in intensity image, Retrieved from https://www.mathworks.com/help/images/ref/edge.html?s_tid=srchti

30. Mathworks, Average or mean of matrix elements, Retrieved from https://www.mathworks.com/help/images/ref/mean2.html?searchHig hlight=mean $\% 20$ of $\% 20$ matrix $\% 20$ elements\&s_tid=doc_srchtitle

31. Mathworks, Standard deviation of matrix elements, Retrieved from https://www.mathworks.com/help/images/ref/std2.html?searchHighl ight=Standard $\% 20$ deviation $\% 20$ of $\% 20$ matrix $\% 20$ elements\&s_tid=d oc_srchtitle

32. Mathworks, Entropy of grayscale image, Retrieved from https://www.mathworks.com/help/images/ref/entropy.html?searchHi ghlight $=$ The $\% 20$ entropy $\% 20$ of $\% 20$ grayscale $\% 20$ images\&s_tid=doc _srchtitle

33. Mathworks, Texture Analysis Using the Gray-Level Co-Occurrence Matrix (GLCM),Retrieved fromhttps://www.mathworks.com/help/ images/texture-analysis-using-the-gray-level-co-occurrence-matrixglcm.html?s_tid=srchtitle

34. Haralick, R. M., Shanmugam, K., \&Dinstein, I. H. (1973). Textura features for image classification. IEEE Transactions on systems, man, and cybernetics, (6), 610-621.

35. Xin, B., Zhang, J., Zhang, R., \& Wu, X. (2017). Color texture classification of yarn-dyed woven fabric based on dual-side scanning and co-occurrence matrix. Textile Research Journal, 87(15), 18831895 .

36. Eldessouki, M., \& Hassan, M. (2015). Adaptive neuro-fuzzy system for quantitative evaluation of woven fabrics' pilling resistance. Expert Systems with Applications, 42(4), 2098-2113. 